\title{
BMJ Open Characteristics, interventions and outcomes of patients with valvular heart disease hospitalised in China: a cross- sectional study
}

\author{
Xinghe Huang (D) , ${ }^{1,2,3}$ Sanket S Dhruva, ${ }^{4}$ Xin Yuan, ${ }^{5}$ Xueke Bai, ${ }^{2}$ Yuan Lu (D) , \\ Xiaofang Yan, ${ }^{2}$ Jiamin Liu, ${ }^{2}$ Wei Li, ${ }^{2}$ Danli Hu, ${ }^{2}$ Runqing $\mathrm{Ji}^{2}$ Min Gao, ${ }^{2}$ \\ Fengyu Miao, ${ }^{2}$ Jiaying Li, ${ }^{2}$ Jinzhuo Ge, ${ }^{2}$ Harlan M Krumholz (D) , ${ }^{6,7,8}$ Jing Li (1) ${ }^{1,2}$
}

To cite: Huang $X$, Dhruva SS, Yuan $\mathrm{X}$, et al. Characteristics, interventions and outcomes of patients with valvular heart disease hospitalised in China: a cross-sectional study. BMJ Open 2021;11:e052946. doi:10.1136/ bmjopen-2021-052946

- Prepublication history and additional supplemental material for this paper are available online. To view these files, please visit the journal online (http://dx.doi.org/10.1136/ bmjopen-2021-052946)

$\mathrm{XH}$ and SSD are joint first authors.

HMK and JL are joint senior authors.

Received 28 April 2021 Accepted 01 October 2021

Check for updates

(C) Author(s) (or their employer(s)) 2021. Re-use permitted under CC BY-NC. No commercial re-use. See rights and permissions. Published by BMJ.

For numbered affiliations see end of article.

Correspondence to

Dr Jing Li; jing.li@fwoxford.org

\section{ABSTRACT}

Objectives Little is known about contemporary characteristics and management of valvular heart disease (VHD) in China. This study aimed to examine the clinical characteristics, aetiology and type of VHD, interventions and in-hospital outcomes of patients with VHD hospitalised in China.

Methods We used a two-stage random sampling design to create a nationally representative sample of patients with VHD hospitalised in 2015 in China and included adult patients with mild, moderate or severe VHD. We abstracted data from medical records, including echocardiogram reports, on patient characteristics, aetiology, type and severity of VHD, interventions and inhospital outcomes. We weighted our findings to estimate nationally representative hospitalisations. We performed multivariable logistic regression analysis to identify factors associated with valve intervention.

Results In 2015, 38841 patients with VHD were hospitalised in 188 randomly sampled hospitals, representing 662384 inpatients with VHD in China. We sampled 9363 patients, mean age 68.7 years $(95 \%$ Cl 42.2 to 95.2$)$ and $46.8 \%(95 \% \mathrm{Cl} 45.8 \%$ to $47.8 \%)$ male, with an echocardiogram. Degenerative origin was the predominant aetiology overall $(33.3 \%, 95 \% \mathrm{Cl}$ $32.3 \%$ to $34.3 \%$ ), while rheumatic origin was the most frequent aetiology among patients with VHD as the primary diagnosis $(37.4 \%, 95 \% \mathrm{Cl} 35.9 \%$ to $38.8 \%)$. Rheumatic origin was also the most common aetiology among patients with moderate or severe VHD $(27.3 \%$, $95 \% \mathrm{Cl} 25.6 \%$ to $29.0 \%$ and $33.6 \%, 95 \% \mathrm{Cl} 31.9 \%$ to $35.2 \%$, respectively). The most common VHD was mitral regurgitation $(79.1 \%, 95 \% \mathrm{Cl} 78.2 \%$ to $79.9 \%)$, followed by tricuspid regurgitation $(77.4 \%, 95 \% \mathrm{Cl} 76.5 \%$ to $78.2 \%$ ). Among patients with a primary diagnosis of severe VHD who were admitted to facilities capable of valve intervention, $35.6 \%$ (95\% Cl $33.1 \%$ to $38.1 \%$ ) underwent valve intervention during the hospitalisation. The likelihood of intervention decreased significantly among patients with higher operative risk.

Conclusions Among patients with VHD hospitalised in China, the predominant aetiology was degenerative in origin; among patients with moderate or severe VHD, rheumatic origin was the most common aetiology. Targeted strategies and policies should be promoted to address
Strengths and limitations of this study

- This is the first study to provide a comprehensive description of features and in-hospital outcomes of patients with valvular heart disease hospitalised in China.

- This study used a two-stage random sampling design to create a nationally representative sample of patients with valvular heart disease hospitalised in China.

- Because this study did not include military hospitals and some randomly sampled hospitals did not have any inpatients with valvular heart disease during the study period, the national numbers of patients with valvular heart disease may be underestimated.

- Patients may have undergone valve intervention at a subsequent hospitalisation in the same hospital or in another hospital, which was not captured by this study.

- This study did not collect enough information to evaluate the risks and benefits of intervention for valvular heart disease for individual patients.

degenerative VHD. Patients with severe VHD may be undertreated, particularly those with high operative risk.

\section{INTRODUCTION}

Valvular heart disease (VHD) is common and associated with increased morbidity and mortality. ${ }^{1}$ Globally, more than 63.7 million people are affected by VHD. ${ }^{2}{ }^{3}$ Rheumatic heart disease (RHD) is the principal cause of VHD in developing countries. ${ }^{1}$ In 2015, approximately 33 million people globally suffered from RHD, resulting in 11 million disability-adjusted life-years. ${ }^{3} \quad$ Additionally, population ageing is leading to an increased global burden of non-rheumatic VHD. ${ }^{2} 4$ Between 1990 and 2017, the Global Burden of Disease Study found the number of people with degenerative VHD doubled globally. ${ }^{2}$ 
As the world's most populous country, China faces challenges of a massive overall burden of VHD. Onefifth of all people with RHD reside in China, and RHD resulted in more than 70000 deaths in 2015. ${ }^{3}$ The ageing of China's population suggests a likely increasing burden of VHD from degenerative aetiology. Meanwhile, advanced interventional techniques have been developed, including transcatheter aortic valve replacement; while this technology has been available since 2010, fewer than 700 patients had received it in China by the end of $2015 .^{5}$ Despite the possible changing epidemiological pattern of VHD and availability of advanced interventional techniques, comprehensive national data about hospitalisations among patients with VHD in China are lacking. Previous studies in China have been from a single centre or province, focused on single aetiology (eg, RHD only), a specific valve (eg, mitral valve disease only) or focused on older patients. ${ }^{6-10}$ These prior studies have shown that rheumatic origin was the predominant aetiology. ${ }^{11}$ In addition, despite the prevalence and relevance to treatment strategies of right-sided $\mathrm{VHD},{ }^{12}$ it has been long ignored and only examined in a handful of international epidemiological studies. ${ }^{10} 1314$ Therefore, there are important questions to be answered to better understand VHD in China, which could ultimately direct efforts to reduce the burden of these treatable diseases. These questions include: how many patients with VHD are hospitalised each year in China; what are comorbidities among these hospitalised patients; what is the aetiology, type and severity of VHD; are there differences by age and sex in aetiology and VHD type; what is the proportion of patients undergoing valve intervention; what factors are associated with valve intervention; and what are in-hospital outcomes of patients with VHD hospitalised in China.

Accordingly, we conducted the China Patient-Centred Evaluative Assessment of Cardiac Events Retrospective Study of VHD (China PEACE-Retrospective VHD Study), drawing a random sample of patients with VHD who were hospitalised and examining their characteristics, aetiology and type of VHD, interventions and in-hospital outcomes through review of in-hospital medical records. Understanding these issues will help refine the focus of future research and ultimately improve clinical outcomes for patients with VHD.

\section{METHODS}

\section{Study design}

Our study is part of the National Studies of Major Chronic Disease in China in 2015. We obtained a nationally representative sample of VHD hospitalisations in 2015 using a two-stage random sampling design, similar to our previous study of myocardial infarction (online supplemental material). ${ }^{15}$ In the first stage, we identified all non-military hospitals in China and excluded prison hospitals, specialised hospitals without a division for cardiovascular disease, and specialised hospitals of traditional Chinese medicine (online supplemental figure S1). The remaining hospitals were stratified into five groups by economic-geographical regions and urbanicity (eastern rural, central rural, western rural, eastern urban and central-western urban) because hospital volumes and clinical capacities differ between urban and rural areas and across Mainland China's three official economic-geographical regions. We grouped the central and western urban regions because incomes and health services capacity per person are similar. In the three rural strata, the sampling framework consisted of the central hospital (the general hospital with the largest clinical capacity) in each of the predefined rural areas. In the two urban strata, the sampling framework consisted of all secondary and tertiary hospitals in each of the predefined urban areas. We did not include primary hospitals, which typically lack capability to diagnose and treat VHD.

In the second stage, we identified all hospitalisations that began in 2015 with a discharge diagnosis of VHD from the sampled hospitals, using each local hospital's database. We then drew hospitalisations using systematic random sampling procedures with prespecified ratios based on annual volume. We identified a discharge diagnosis of VHD by searching International Classification of Diseases-Clinical Modification codes 10 (ICD-10-CM) (I05.x, I06.x, I07.x, I08.x, I09.801, I09.802, I33.x, I34.x, I35.x, I36.x, I37.x or I38.x); when ICD-10-CM codes were unavailable, we searched diagnosis terms in the medical record (details in online supplemental material).

We obtained an electronic copy of the medical record of each sampled hospitalisation for data abstraction. To ensure accuracy, data were centrally and independently abstracted using standardised definitions by two trained and certified abstractors. Any disagreement was resolved through review of the original medical records. Additionally, clinicians at the coordinating centre compared randomly selected records and abstractions to verify data accuracy.

\section{Study population}

We included adult patients (aged $\geq 18$ years) with a discharge diagnosis of VHD, either as the primary diagnosis or as a comorbidity (secondary diagnosis). Patients with congenital VHD or with an uncertain diagnosis (ie, without an echocardiogram during the selected hospitalisation or without defined severity) were excluded (online supplemental figure S1). If a patient had multiple hospitalisations for VHD during 2015, we included only data from the first hospitalisation. Therefore, only one hospitalisation for each patient was included in this study.

\section{Data elements and definitions}

We abstracted data on patient characteristics, including age, sex and both cardiac and non-cardiac comorbidities. We defined comorbidities based on discharge diagnosis terms. We also abstracted types, potential aetiologies, severities of valvular lesions and details of echocardiographic findings. 
VHD types were mitral stenosis, mitral regurgitation, aortic stenosis, aortic regurgitation, tricuspid stenosis, tricuspid regurgitation, pulmonary stenosis and pulmonary regurgitation. Multiple VHD was defined as moderate or severe valvular lesions occurring on $\geq 2$ valves. ${ }^{16}{ }^{17}$ Mixed VHD was defined as the combination of stenotic and regurgitant lesions on the same valve. ${ }^{16}$ We first determined VHD type by ICD-10-CM codes or discharge diagnosis terms (details in online supplemental material). If the diagnosis did not indicate which valve was affected, cardiologists at the coordinating centre reviewed the echocardiogram report to obtain this information.

VHD severities were classified as mild, moderate, and severe; these were determined by the discharge diagnosis, if the discharge diagnosis was unavailable, then parameters of the echocardiogram report, according to definitions in clinical practice guidelines (quantitative criteria in online supplemental material), were used to determine severity. ${ }^{1819}$ We further verified the accuracy of the VHD severity abstracted from the discharge diagnosis with echocardiogram reports; overall, consistency was $95 \%$. Among patients who received multiple echocardiograms during the hospitalisation, we included only the first echocardiogram. Aetiologies of VHD were determined according to discharge diagnosis or echocardiographic findings (criteria in online supplemental material).

We defined a hospital as having the capability to perform valve intervention if at least one sampled patient from that hospital underwent valve intervention (either surgical or percutaneous) during the study period. These interventions included valve repair and replacement. The in-hospital outcomes were length of hospital stay and death.

\section{Statistical analysis}

To estimate the number of patients with VHD hospitalised nationally across China, we applied weights proportional to the inverse sampling fraction of hospitals within each stratum and the sampling fraction of patients within each hospital, similar to prior studies. ${ }^{15}$ We used the same weighting method to obtain nationally representative descriptive measures from our study sample. Normal distributed continuous variables are presented as means with $95 \%$ CIs, skewed distributed continuous variables are presented as medians with IQRs, and categorical variables are presented as percentages with $95 \%$ CIs. Comparisons between categorical variables were performed using the $\chi^{2}$ test; comparisons between normal distributed continuous variables were performed using the Student's t-test or the one-way analysis of variance; comparisons between skewed distributed continuous variables were performed using the Mann-Whitney U test or the Kruskal-Wallis test. We compared the characteristics between patients with VHD as the primary diagnosis and as a secondary diagnosis, and among patients with different VHD severities and VHD types. We calculated the proportion of each aetiology and VHD type among all patients, and by age and sex. We calculated the age-specific and aetiology-specific rate of hospitalisation as a weighted estimated number of patients with VHD hospitalised divided by the total population in the specific age group. ${ }^{20}$ Among patients admitted to hospitals with the capability to perform valve interventions, we compared in-hospital outcomes between patients with and without valve intervention, and between patients with VHD as their primary diagnosis and those with VHD as a secondary diagnosis. We further performed multivariable logistic regression analysis among patients with VHD as the primary diagnosis to identify factors associated with valve intervention. A twosided $p<0.05$ was considered statistically significant. Statistical analyses were performed using SAS software (V.9.4; SAS Institute).

\section{Patient and public involvement}

Patients and/or the public were not involved in the design, or conduct, or reporting, or dissemination plans of this research.

\section{RESULTS}

Among the 205 sampled hospitals, 8 had no VHD hospitalisations in 2015 and 9 declined to participate. From the remaining 188 hospitals (online supplemental figure S2), we identified 38841 hospitalisations with a discharge diagnosis of VHD in 2015. After applying sampling weights, these patients represented 662384 patients with VHD who were hospitalised in 2015 in China.

\section{Patient characteristics}

Of the 38841 hospitalisations, we sampled 13338 hospitalisations and obtained medical records for 13138 hospitalisations (98.5\%) (online supplemental figure S1). After excluding 3534 hospitalisations with an uncertain diagnosis, 60 hospitalisations with congenital VHD and 181 rehospitalisations for the same patient, our final study sample for analysis included 9363 patients with VHD who were hospitalised in 2015 and for whom echocardiogram results were available. Patient mean age was 68.7 years (95\% CI 42.2 to 95.2$)$ and $46.8 \%$ (95\% CI $45.8 \%$ to $47.8 \%$ ) were male (table 1 ). Heart failure $(59.7 \%, 95 \%$ CI $58.7 \%$ to $60.6 \%)$, atrial fibrillation $(33.0 \%, 95 \%$ CI $32.0 \%$ to $33.9 \%)$, hypertension $(37.6 \%$, 95\% CI $36.7 \%$ to $38.6 \%)$ and coronary heart disease $(23.9 \%, 95 \%$ CI $23.0 \%$ to $24.8 \%$ ) were the most common comorbidities and $4.8 \%$ (95\% CI $4.4 \%$ to $5.2 \%$ ) of patients had undergone previous valve intervention.

There were $5404(57.7 \%)$ patients with VHD as their primary diagnosis, representing 211526 hospitalised patients with VHD as the primary diagnosis in 2015 in China. Compared with patients for whom VHD was the secondary diagnosis, those with VHD as the primary diagnosis were younger; more likely to have heart failure, atrial fibrillation, pulmonary hypertension and previous valve intervention; and less likely to have hypertension, coronary heart disease, chronic obstructive pulmonary disease and dyslipidaemia (table 1). The proportion of 
Table 1 Characteristics of patients with valvular heart disease (VHD) hospitalised in China

\begin{tabular}{|c|c|c|c|c|}
\hline & Total $(n=9363)$ & $\begin{array}{l}\text { Primary diagnosis of } \\
\text { VHD }(n=5404)\end{array}$ & $\begin{array}{l}\text { Secondary diagnosis of } \\
\text { VHD }(n=3959)\end{array}$ & $P$ value \\
\hline \multicolumn{5}{|l|}{ Demographics } \\
\hline Age, median (IQR), years & $71(60-79)$ & $65(56-75)$ & $75(65-81)$ & $<0.001$ \\
\hline Age, mean $(95 \% \mathrm{Cl})$, years & 68.7 (42.2 to 95.2$)$ & 64.8 (41.3 to 88.3$)$ & $72.2(44.2$ to 100.2$)$ & $<0.001$ \\
\hline Age, \% (95\% Cl) & & & & $<0.001$ \\
\hline $18-44$ years & 5.0 (4.6 to 5.5$)$ & 6.8 (6.1 to 7.6$)$ & 3.4 (2.9 to 3.9 ) & \\
\hline $45-54$ years & 10.6 (10.0 to 11.2$)$ & 15.1 (14.0 to 16.2$)$ & 6.6 (5.9 to 7.3$)$ & \\
\hline $55-64$ years & 19.2 (18.4 to 20.0$)$ & 25.5 (24.2 to 26.8 ) & 13.6 (12.6 to 14.6$)$ & \\
\hline $65-74$ years & 26.0 (25.2 to 26.9$)$ & 25.9 (24.6 to 27.2$)$ & 26.2 (25.0 to 27.4$)$ & \\
\hline$\geq 75$ years & 39.0 (38.1 to 40.0$)$ & 26.6 (25.3 to 27.9$)$ & 50.1 (48.7 to 51.5$)$ & \\
\hline Male, \% $(95 \% \mathrm{Cl})$ & 46.8 (45.8 to 47.8 ) & 43.2 (41.8 to 44.7$)$ & 50.0 (48.6 to 51.4$)$ & $<0.001$ \\
\hline \multicolumn{5}{|l|}{ Comorbidities, \% (95\% Cl) } \\
\hline Heart failure & 59.7 (58.7 to 60.6$)$ & 76.1 (74.8 to 77.4$)$ & 45.0 (43.6 to 46.4$)$ & $<0.001$ \\
\hline Atrial fibrillation & 33.0 (32.0 to 33.9) & 45.2 (43.7 to 46.7$)$ & 22.0 (20.9 to 23.2) & $<0.001$ \\
\hline Pulmonary hypertension & 10.7 (10.1 to 11.4$)$ & 13.0 (12.0 to 14.0$)$ & 8.7 (7.9 to 9.5$)$ & $<0.001$ \\
\hline Hypertension & 37.6 (36.7 to 38.6$)$ & 32.2 (30.8 to 33.6 ) & 42.5 (41.2 to 43.9$)$ & $<0.001$ \\
\hline Coronary heart disease & 23.9 (23.0 to 24.8$)$ & 22.6 (21.4 to 23.9 ) & 25.1 (23.9 to 26.3 ) & 0.006 \\
\hline Chronic obstructive pulmonary disease & 14.6 (13.9 to 15.3$)$ & 9.4 (8.5 to 10.3$)$ & 19.3 (18.2 to 20.4$)$ & $<0.001$ \\
\hline Dyslipidaemia & 10.6 (10.0 to 11.3$)$ & 8.1 (7.3 to 9.0$)$ & 12.9 (12.0 to 13.9$)$ & $<0.001$ \\
\hline Type two diabetes & 9.7 (9.1 to 10.3$)$ & 9.4 (8.5 to 10.3$)$ & $9.9(9.1$ to 10.8$)$ & 0.366 \\
\hline Previous valve intervention, \% (95\% Cl) & 4.8 (4.4 to 5.2$)$ & 7.5 (6.8 to 8.4$)$ & 2.3 (1.9 to 2.8$)$ & $<0.001$ \\
\hline Aetiology, \% (95\% Cl) & & & & $<0.001$ \\
\hline Degenerative & 33.3 (32.3 to 34.3 ) & 15.2 (14.2 to 16.3 ) & 49.4 (48.0 to 50.8$)$ & \\
\hline Rheumatic & 22.2 (21.4 to 23.1$)$ & 37.4 (35.9 to 38.8$)$ & 8.7 (7.9 to 9.5$)$ & \\
\hline Ischaemic & 3.1 (2.8 to 3.5$)$ & $3.2(2.7$ to 3.8$)$ & $3.0(2.5$ to 3.5$)$ & \\
\hline Infective & $1.0(0.8$ to 1.2$)$ & 1.8 (1.4 to 2.2$)$ & $0.2(0.1$ to 0.4$)$ & \\
\hline Combined & 12.5 (11.8 to 13.2$)$ & 14.0 (13.0 to 15.0$)$ & $11.2(10.3$ to 12.1$)$ & \\
\hline Unknown & 27.9 (27.0 to 28.9$)$ & 28.4 (27.1 to 29.8$)$ & 27.5 (26.2 to 28.8$)$ & \\
\hline Disease type, \% (95\% Cl) & & & & $<0.001$ \\
\hline Single mitral valve disease & $16.9(16.1$ to 17.6$)$ & 20.7 (19.5 to 21.9$)$ & 13.4 (12.5 to 14.4$)$ & \\
\hline Single aortic valve disease & $13.8(13.1$ to 14.5$)$ & $12.7(11.7$ to 13.7$)$ & 14.8 (13.8 to 15.8$)$ & \\
\hline Single tricuspid valve disease & 10.1 (9.5 to 10.8$)$ & 9.0 (8.2 to 9.9$)$ & 11.2 (10.3 to 12.1$)$ & \\
\hline Multiple valve disease & 29.5 (28.6 to 30.4$)$ & 41.1 (39.6 to 42.5 ) & 19.1 (18.0 to 20.3$)$ & \\
\hline Other & 29.7 (28.8 to 30.7$)$ & $16.6(15.5$ to 17.7$)$ & 41.5 (40.1 to 42.9$)$ & \\
\hline
\end{tabular}

Single mitral valve disease includes mitral stenosis or mitral regurgitation; single aortic valve disease includes aortic stenosis or aortic regurgitation; single tricuspid valve disease includes tricuspid stenosis or tricuspid regurgitation; other includes mixed aortic valve disease, mixed mitral valve disease, single pulmonary disease and other combination of two or more mild valve disease.

VHD, valvular heart disease.

patients with heart failure, atrial fibrillation and pulmonary hypertension increased with VHD severity (online supplemental table S1). Detailed comparisons of patient characteristics across VHD types are shown in online supplemental table S2.

\section{Aetiologies of VHD}

Overall, $33.3 \%$ (95\% CI $32.3 \%$ to $34.3 \%$ ) of patients with VHD had degenerative origin and 22.2\% (95\% CI 21.4\% to $23.1 \%$ ) had rheumatic origin (figure 1A). Rheumatic origin was the most frequent aetiology among patients with VHD as the primary diagnosis $(37.4 \%, 95 \%$ CI $35.9 \%$ to $38.8 \%$ ) and degenerative origin was the predominant aetiology among patients with VHD as the secondary diagnosis $(49.4 \%, 95 \%$ CI $48.0 \%$ to $50.8 \%$ ) (table 1 ). Degenerative origin was the most predominant aetiology in both sexes: $36.0 \%$ (95\% CI $34.5 \%$ to $37.4 \%$ in males 
A
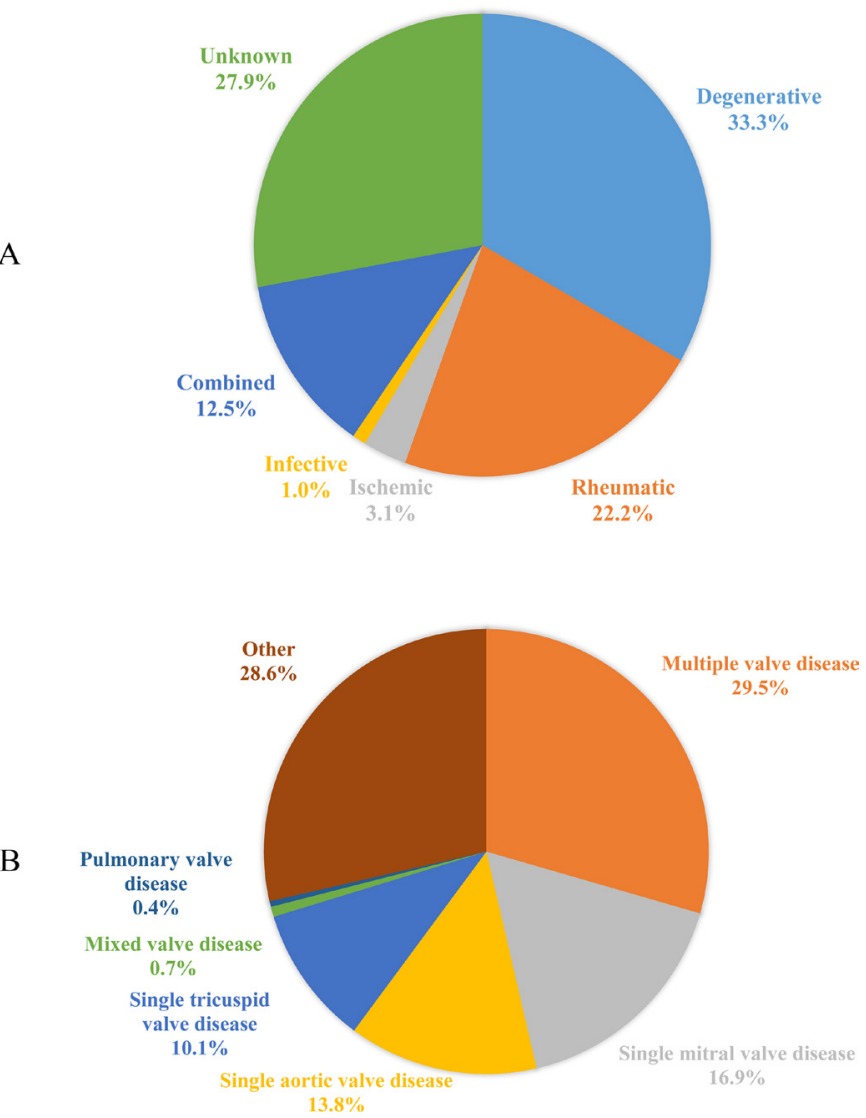

Figure 1 Aetiology and type of valvular heart disease among patients hospitalised in China.

and $30.9 \%$ (95\% CI $29.7 \%$ to $32.3 \%$ ) in females (online supplemental figure S3). Rheumatic origin was more common in females $(28.7 \%, 95 \%$ CI $27.5 \%$ to $30.0 \%)$ than males $(14.8 \%, 95 \%$ CI $13.8 \%$ to $15.9 \%)$. Among patients with moderate or severe $\mathrm{VHD}$, rheumatic origin was the most common aetiology $(27.3 \%, 95 \%$ CI $25.6 \%$ to $29.0 \%$ and $33.6 \%, 95 \%$ CI $31.9 \%$ to $35.2 \%$, respectively).

When examining age-specific hospitalisation rates for VHD, the peak rates of RHD were among patients aged 65-74 years, with a decrease with younger age. Rates of infective VHD were low across ages; while rates of other aetiologies increased with age (figure 2 and online supplemental tables S3 and S4). Similarly, among all patients with VHD, the proportion of patients with degenerative aetiology increased with age, from $6.1 \%$ (95\% CI $4.1 \%$ to $8.6 \%)$ among patients $<45$ years to $56.6 \%$ (95\% CI $55.0 \%$ to $58.2 \%$ ) in those $\geq 75$ years; the proportion who had RHD was $31.0 \%$ (95\% CI $26.8 \%$ to $35.4 \%$ ) among patients <45 years, $44.1 \%$ (95\% CI $40.9 \%$ to $47.2 \%$ ) in 45-54 years and then decreased with age (online supplemental figure S4). Aetiologies stratified by VHD type are presented in online supplemental figure S5.

\section{Types and severities of VHD}

Among all patients with VHD hospitalised in China, mitral regurgitation was the most frequent type of VHD $(79.1 \%$, 95\% CI $78.2 \%$ to $79.9 \%$; $44.1 \%, 95 \%$ CI $43.1 \%$ to $45.0 \%$ with mild severity), followed by tricuspid regurgitation
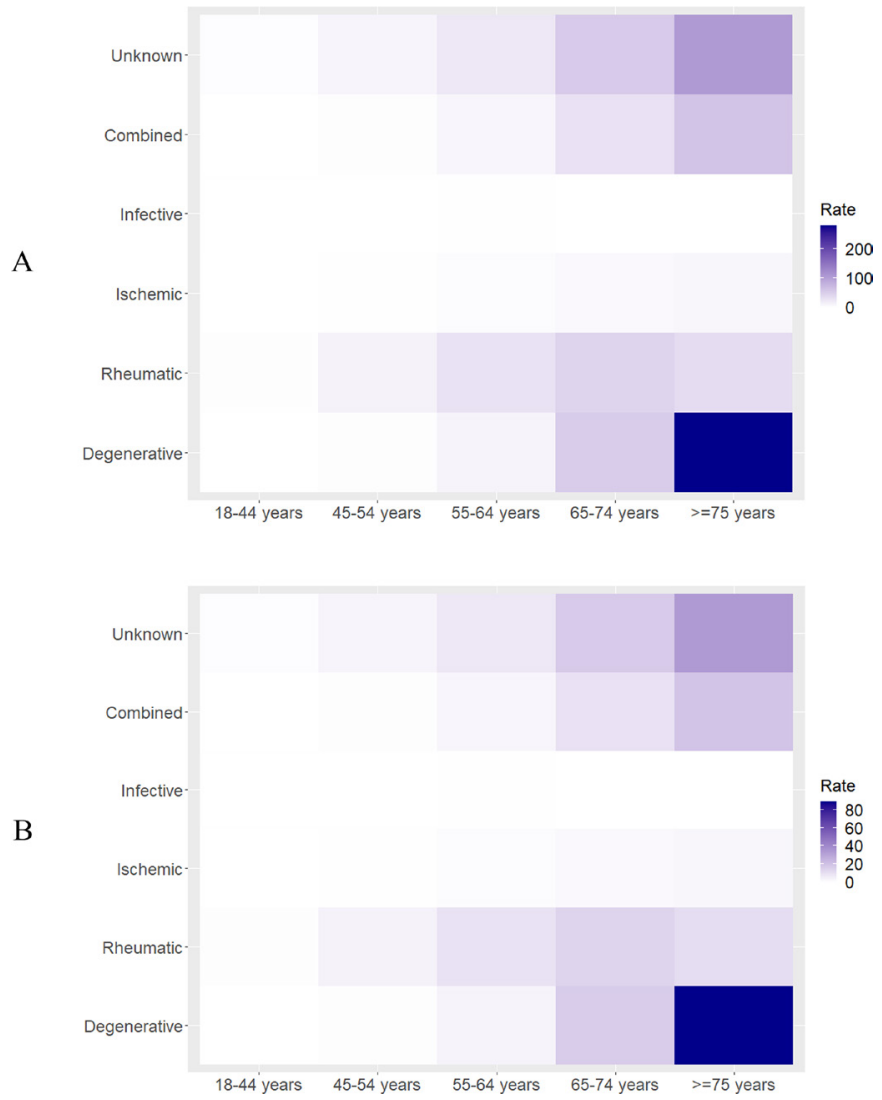

Figure 2 Rate of hospitalisation with valvular heart disease in age-specific and aetiology-specific groups (cases per 100 000). The age-specific and aetiology-specific rate of hospitalisation was the weighted estimated number of patients with valvular heart disease hospitalised divided by the total population in the specific age group (A) among all patients hospitalised with valvular heart disease; (B) among patients hospitalised with valvular heart disease as the primary diagnosis. Darker colour represents higher rates.

(77.4\%, $95 \%$ CI $76.5 \%$ to $78.2 \%$ ); $45.8 \%$ (95\% CI $44.8 \%$ to $46.8 \%$ ) with mild severity) and aortic regurgitation (70.6\% (95\% CI $69.7 \%$ to $71.6 \%$ ); $47.7 \%$ (95\% CI $46.7 \%$ to $48.7 \%$ with mild severity) (table 2 ).

Overall, $29.5 \%$ (95\% CI $28.6 \%$ to $30.4 \%$ ) of patients with VHD hospitalised had multiple VHD (figure 1B). The proportion of multiple VHD was higher among patients with VHD as the primary diagnosis compared with patients with VHD as the secondary diagnosis $(41.1 \%$ vs $19.1 \%, \mathrm{p}<0.001$ ) (table 1 ). The most frequent combination of multiple VHD was mitral regurgitation/tricuspid regurgitation, followed by aortic regurgitation/tricuspid regurgitation and mitral regurgitation/aortic regurgitation (online supplemental table S5). Data on VHD type by sex and age are presented in online supplemental figures S6 and S7.

\section{Interventions for VHD}

Thirty-eight hospitals included in our study had capability to perform valve intervention. Among patients with a primary diagnosis of mild, moderate and severe VHD who were hospitalised at these facilities during our study 
Table 2 Types and severities of valvular heart disease

\begin{tabular}{|c|c|c|c|c|}
\hline & Total, \% (95\% Cl) & Mild, \% (95\% Cl) & Moderate, \% (95\% Cl) & Severe, \% $(95 \% \mathrm{Cl})$ \\
\hline Mitral regurgitation & 79.1 (78.2 to 79.9$)$ & 44.1 (43.1 to 45.0 ) & 17.2 (16.5 to 18.0$)$ & 15.6 (14.8 to 16.3$)$ \\
\hline Mitral stenosis & 19.6 (18.8 to 20.5$)$ & 5.7 (5.3 to 6.2) & 8.0 (7.5 to 8.5$)$ & 5.4 (4.9 to 5.9$)$ \\
\hline Aortic stenosis & 14.8 (14.1 to 15.6$)$ & 6.3 (5.8 to 6.8$)$ & 4.1 (3.7 to 4.5$)$ & 4.1 (3.7 to 4.5$)$ \\
\hline Tricuspid regurgitation & 77.4 (76.5 to 78.2$)$ & 45.8 (44.8 to 46.8 ) & 16.1 (15.4 to 16.9$)$ & 13.3 (12.6 to 14.0$)$ \\
\hline Pulmonary regurgitation & 12.9 (12.2 to 13.6$)$ & $11.0(10.4$ to 11.7$)$ & 1.3 (1.1 to 1.6$)$ & 0.2 (0.1 to 0.3$)$ \\
\hline Pulmonary stenosis & $0.4(0.3$ to 0.5$)$ & 0.1 (0.1 to 0.2$)$ & 0.1 (0.1 to 0.2$)$ & 0.1 (0.1 to 0.2$)$ \\
\hline
\end{tabular}

period, $5.5 \%$ (95\% CI $3.3 \%$ to $8.5 \%), 21.0 \%$ (95\% CI $18.2 \%$ to $24.0 \%$ ) and $35.6 \%$ (95\% CI $33.1 \%$ to $38.1 \%$ ) underwent valve intervention during the index hospitalisation, respectively (figure 3$)$. Only four patients $(2.2 \%)$ underwent valve intervention during a rehospitalisation within the study period. For mitral and aortic valve disease, most patients underwent valve replacement; for tricuspid valve disease, most patients underwent valve repair (table 3 ).

Compared with those younger than 45 years, older patients (OR $0.58,95 \%$ CI 0.42 to 0.80 for age $45-54$ years; OR $0.35,95 \%$ CI 0.25 to 0.48 for age $55-64$ years; OR 0.10 , $95 \%$ CI 0.07 to 0.14 for age $65-74$ years; OR $0.01,95 \%$ CI 0.01 to 0.03 for age $\geq 75$ years) were less likely to undergo valve intervention. Patients with mild (OR 0.12, 95\% CI 0.08 to 0.20 ) or moderate (OR $0.43,95 \%$ CI 0.34 to 0.54 ) VHD severity were less likely to undergo valve intervention compared with those with severe VHD. Hypertension (OR $0.71,95 \%$ CI 0.53 to 0.93 ), type 2 diabetes (OR $0.44,95 \%$ CI 0.27 to 0.72 ), chronic obstructive pulmonary disease (OR $0.41,95 \%$ CI 0.22 to 0.77 ) and previous valve intervention (OR $0.65,95 \%$ CI 0.42 to 0.99 ) were associated with lower likelihood of valve intervention. In

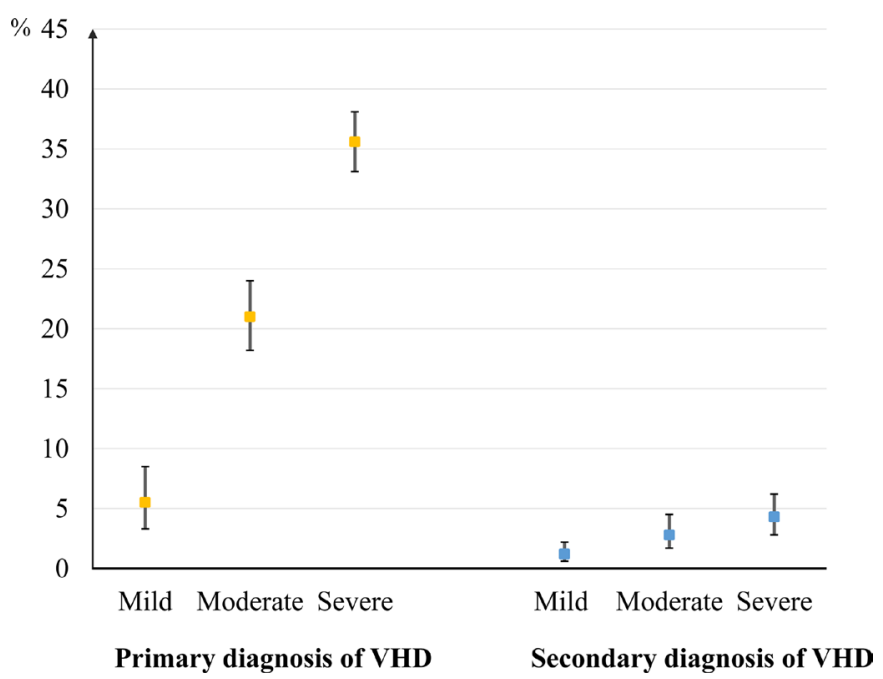

Figure 3 Proportion of patients hospitalised with valvular heart disease (VHD) in hospitals capable of valve intervention who underwent intervention. Each square represents the point estimate, and the line shows the $95 \% \mathrm{Cl}$. contrast, patients with pulmonary hypertension (OR 1.69, $95 \%$ CI 1.29 to 2.23 ) were more likely to undergo valve intervention (figure 4).

\section{In-hospital outcomes}

Among 4182 patients hospitalised at facilities with capability to perform valve intervention, the median length of hospital stay was 10 days (IQR 7-16) and in-hospital death was $1.3 \%$ (95\% CI $1.0 \%$ to $1.7 \%$ ) (online supplemental table S6). The median length of hospital stay among patients with and without valve intervention was 24 days (IQR 18\%32) and 9 days (IQR 6\%13), respectively $(\mathrm{p}<0.001)$. In-hospital death occurred in $1.4 \%$ (95\% CI $0.7 \%$ to $2.5 \%)$ and $1.3 \%$ (95\% CI $1.0 \%$ to $1.7 \%)$ of patients who underwent and did not undergo valve intervention, respectively $(\mathrm{p}=0.862)$. Details of in-hospital outcomes between patients with VHD as the primary diagnosis and as the secondary diagnosis are shown in online supplemental table S7.

\section{DISCUSSION}

This nationally representative study provides the first comprehensive picture of patients with VHD who are hospitalised in China, including data on patient characteristics, aetiology and type, interventions and in-hospital outcomes. We estimate that more than 662384 patients with VHD were hospitalised in China in 2015. We are the first to find that degenerative origin was the most frequent aetiology among patients with VHD hospitalised in China, compared with previous single-centre studies, which have shown that rheumatic valve disease was the predominant aetiology. ${ }^{621}$ However, among patients with VHD as the primary diagnosis and among patients with moderate or severe VHD, rheumatic valve disease was the predominant aetiology. The most common VHD was mitral regurgitation, followed by tricuspid regurgitation; almost one-third of patients suffered from moderate or severe tricuspid regurgitation. Among patients with a primary diagnosis of severe VHD who were hospitalised at facilities capable of valvular intervention, only one-third underwent valve intervention during the hospitalisation, with lower intervention rates among those with advanced age, more comorbidities or previous valve intervention. 
Table 3 Intervention types among patients who underwent valve intervention during hospitalisation

\begin{tabular}{llcl}
\hline & $\begin{array}{l}\text { Valve replacement, } \\
\%(95 \% \mathrm{Cl})\end{array}$ & $\begin{array}{l}\text { Valve repair, } \\
\%(95 \% \mathrm{Cl})\end{array}$ & $\begin{array}{l}\text { Percutaneous valve intervention, } \\
\%(95 \% \mathrm{Cl})\end{array}$ \\
\hline Mitral valve $(\mathrm{n}=488)$ & $81.1(77.6$ to 84.3$)$ & $17.3(14.3$ to 20.8$)$ & $1.5(0.7$ to 2.9$)$ \\
Aortic valve $(\mathrm{n}=248)$ & $97.3(94.6$ to 98.9$)$ & $2.7(1.1$ to 5.4$)$ & - \\
\hline Tricuspid valve $(\mathrm{n}=211)$ & $6.0(3.4$ to 9.8$)$ & $94.0(90.2$ to 96.6$)$ & - \\
Pulmonary valve $(\mathrm{n}=6)$ & - & $79.0(40.2$ to 97.8$)$ & $21.0(2.2$ to 59.8$)$ \\
\hline
\end{tabular}

This is the first study of patients with VHD hospitalised in China using nationally representative data. When including all VHD (including mild), we found that degenerative valve disease is the predominant aetiology. In the USA and Europe in recent decades, RHD incidence has fallen substantially, and degenerative VHD has replaced RHD as the leading cause of VHD. ${ }^{1722}$ Given the increase in life expectancy and the current lack of validated prevention strategies, the burden of degenerative VHD has increased in Asia-Pacific countries as well. ${ }^{123}$ We found more than one-third of patients with VHD hospitalised in China had a degenerative origin of their VHD. This proportion reached three-fifths among patients aged $\geq 75$ years, extending findings from a cohort of older VHD patients in China. ${ }^{9}$ China's ageing population means degenerative VHD prevalence will continue increasing in the future. ${ }^{24}{ }^{25}$ Although most degenerative VHD was mild, $38.4 \%$ was moderate or severe. Some mild degenerative VHD, such as mild aortic stenosis, may progress to severe VHD. Thus, given the high burden of degenerative VHD, more attention should be paid to detection and mitigation in order to reduce adverse clinical consequences. RHD usually begins during childhood as the long-term sequela of acute rheumatic fever. ${ }^{26}$ Although RHD was the dominant aetiology among patients with VHD as the primary diagnosis and when limiting to patients with moderate or severe VHD, we found the rate of hospitalisation with RHD substantially decreased with younger age, supporting RHD prevalence has decreased in China. This decrease is likely related to improvements in prevention and treatment of acute rheumatic fever over the past decades through improved socioeconomic status, living conditions and antibiotic prophylaxis, ${ }^{25}$ and the Global Burden of Disease Study showed death from RHD in China decreased by $47 \%$ from 1990 to $2016 .^{27}$ Because there is still a high burden of RHD, prevention strategies and effective care of affected patients should still be strengthened in China.

To the best of our knowledge, our study is the first to document details about tricuspid regurgitation among patients with VHD hospitalised in China. Nearly half of patients with tricuspid regurgitation had moderate or severe severity. Tricuspid valve disease is associated with decreased long-term survival, ${ }^{28}{ }^{29}$ however, it has received less attention than left-sided VHD and has often been excluded from epidemiological studies of VHD. ${ }^{172130}$ For these reasons, the tricuspid valve is often referred to as the 'forgotten valve'. ${ }^{31}$ The management recommendations in current clinical practice guidelines for tricuspid valve disease lack evidence from rigorous randomised controlled trials; ${ }^{19}{ }^{32}$ thus, there is an urgent need for further prospective research on this topic given the high burden of tricuspid valve disease found in our study. We found that mitral regurgitation was the most common VHD among patients with VHD hospitalised in China, in line with the results of a cohort of older adults with VHD in China. ${ }^{33}$ Similar to our study, a US population-based study found the most frequent VHD was mitral regurgitation, while in Europe, aortic stenosis was the most frequent. $^{2230}$

There may be undertreatment with valve repair or replacement among patients hospitalised at facilities with the capability to perform valve interventions. Only one-third of patients with a primary diagnosis of severe VHD underwent valve intervention, and just an additional $2.2 \%$ later in the year within the same hospital. Undertreatment of VHD has been reported in other international studies. ${ }^{17}$ 34-37 The decision to perform valve intervention is complex and encompasses many factors including life expectancy, comorbidities, surgical risk, feasibility of successful intervention and patient preferences. ${ }^{9} 303839$ We found the likelihood of intervention decreased among patients with high operative risk, including older age, more comorbidities or previous valve intervention. $^{3038} 40$ The low rate of valve intervention for patients with VHD suggests a need for better promotion of both traditional surgical and advanced interventional techniques. Although less invasive valve therapies such as transcatheter aortic valve replacement and transcatheter mitral valve repair were at an early stage in 2015 , mainly available in very large medical centres in China, ${ }^{41}$ there may be opportunities to use these interventions in appropriately selected patients who meet evidence-based criteria. Enhancing multidisciplinary collaboration for VHD may offer potential to reduce the burden of VHD and its clinical sequelae.

\section{Limitations}

This study should be considered in the context of multiple limitations. First, our study was based on data collected in 2015; however, it is the first to provide a comprehensive national picture of patients with VHD hospitalised in China. Second, we may underestimate the national numbers of patients with VHD for the following two reasons: (1) we did not include military hospitals which also provide care for VHD because these data are 


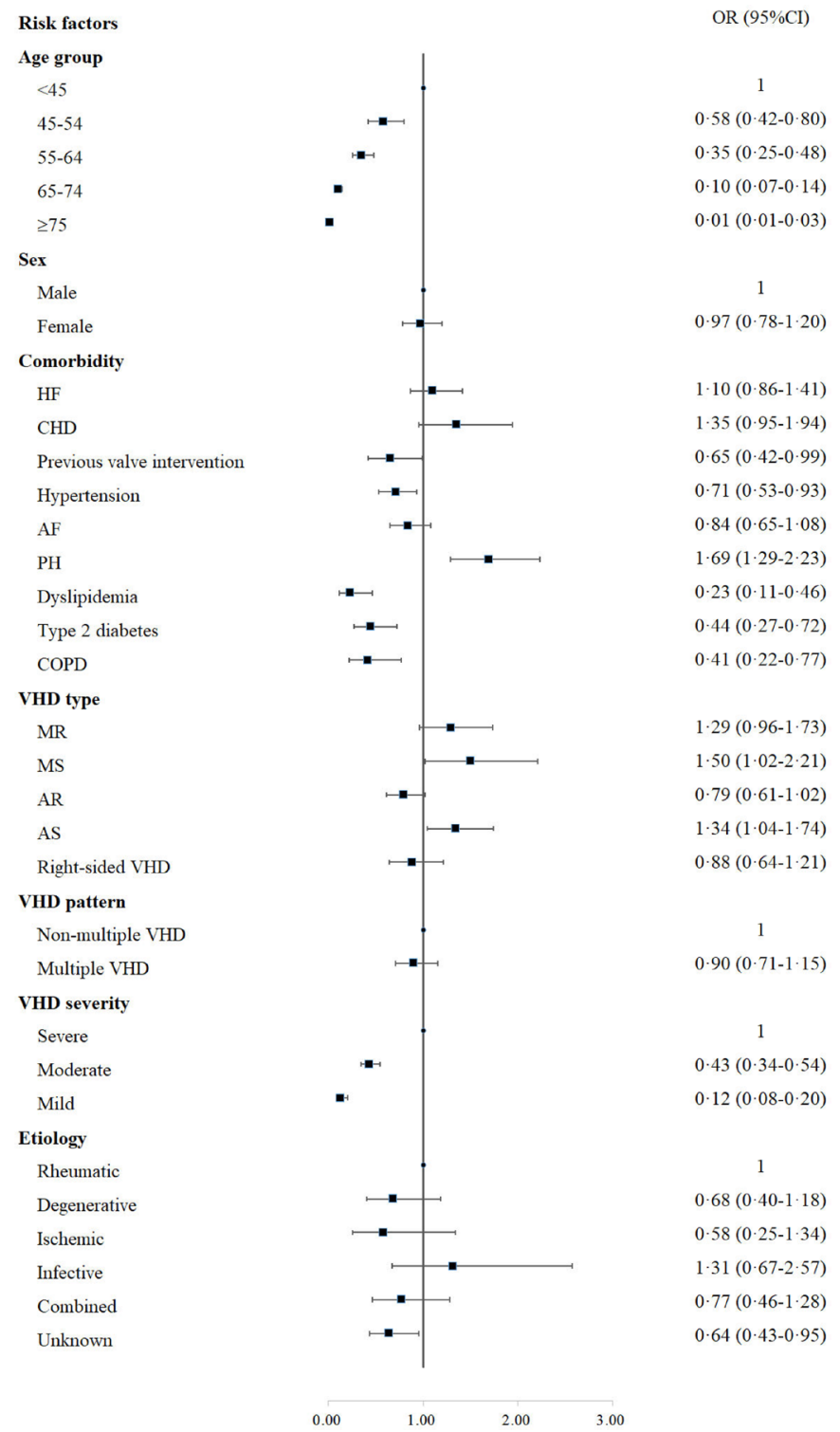

Figure 4 Factors associated with intervention during hospitalisation. The strength of effect is shown along the horizontal axis, with the vertical line demarcating an OR of 1 (ie, no association); estimates to the right (ie, >1) are associated with greater likelihood of intervention, while those to the left (ie, <1) indicate association with less likelihood of intervention. Each square represents the point estimate of the effect of that variable in the model, while the line shows the $95 \% \mathrm{Cl}$. AF, atrial fibrillation; AR, aortic regurgitation; AS, aortic stenosis; CHD, coronary heart disease; COPD, chronic obstructive pulmonary disease; HF, heart failure; $\mathrm{MR}$, mitral regurgitation; MS, mitral stenosis; $\mathrm{PH}$, pulmonary hypertension; VHD, valvular heart disease.

unavailable; (2) some randomly sampled hospitals did not have any inpatients with VHD during the study period, which suggests they may lack capability of diagnosing VHD. Third, we only obtained information from medical records; thus, for approximately $27.9 \%$ of patients, we could not identify aetiology of VHD. Fourth, patients may have undergone valve intervention at a subsequent hospitalisation in the same hospital or in another hospital, which was not captured by our study. However, we were able to account for rehospitalisations at the same hospital in 2015 and only $2 \%$ were rehospitalised and a total of four patients underwent valve intervention at the subsequent hospitalisation in the same hospital during the study period. Fifth, we did not collect enough information to evaluate the risks and benefits of intervention for VHD for individual patients; however, the small proportion of patients undergoing valve intervention likely indicates some degree of undertreatment of VHD.

\section{CONCLUSION}

This contemporary study provides the first comprehensive description of patient characteristics, aetiology, disease features, interventions and in-hospital outcomes of patients with VHD hospitalised in China in 2015. Degenerative origin is the dominant aetiology of VHD. Mitral regurgitation is the most frequent VHD, followed by tricuspid regurgitation; nearly one-third of VHD patients have moderate or severe tricuspid regurgitation. Patients with severe VHD may be undertreated, especially those with high operative risk, which calls for the use of surgical and interventional techniques among patients who meet evidence-based criteria. These findings highlight the opportunities to improve care and outcomes for patients with VHD in China.

\section{Author affiliations}

${ }^{1}$ Fuwai Hospital, Chinese Academy of Medical Sciences, Shenzhen, People's Republic of China

${ }^{2}$ National Clinical Research Center for Cardiovascular Diseases, Fuwai Hospital, Chinese Academy of Medical Sciences and Peking Union Medical College, National Center for Cardiovascular Diseases, Beijing, People's Republic of China

${ }^{3}$ School of Nursing, Peking Union Medical College, Beijing, People's Republic of China

${ }^{4}$ Department of Medicine, University of California, San Francisco School of Medicine and Section of Cardiology, San Francisco Veterans Affairs Medical Center, San Francisco, California, USA

${ }^{5}$ Department of Cardiovascular Surgery, State Key Laboratory of Cardiovascular Disease, Fuwai Hospital, Chinese Academy of Medical Science and Peking Union Medical College, National Center for Cardiovascular Diseases, Xicheng District, People's Republic of China

${ }^{6}$ Center for Outcomes Research and Evaluation, Yale-New Haven Hospital, and Department of Internal Medicine, Yale University School of Medicine, New Haven, Connecticut, USA

${ }^{7}$ Department of Health Policy and Management, Yale University School of Public Health, New Haven, Connecticut, USA

${ }^{8}$ Section of Cardiovascular Medicine, Department of Internal Medicine, Yale University School of Medicine, New Haven, Connecticut, USA

\section{Twitter Harlan M Krumholz @hmkyale}

Acknowledgements We appreciate the multiple contributions made by project teams at the China National Clinical Research Centre for Cardiovascular Diseases for data collection. We are grateful for the funding support provided by Chinese government.

Contributors $\mathrm{JL}$ conceived the study and took the responsibility for the overall content as guarantor. XHH and SSD wrote the first draft of the article. JL, HMK, XY, YL, XFY, JML, WL, DLH, RQJ, MG, FYM, JYL, and JZG helped shape the manuscript. XKB did the statistical analysis, with support from XHH. All authors interpreted the data and approved the final version of the article.

Funding This work was supported by the National Key Research and Development Programme (2018YFC1312400, 2018YFC1312401, 2018YFC1312404) from the 
Ministry of Science and Technology of China and the National Key Technology R\&D Programme (2015BAl12B02) from the Ministry of Science and Technology of China.

Map disclaimer The depiction of boundaries on this map does not imply the expression of any opinion whatsoever on the part of BMJ (or any member of its group) concerning the legal status of any country, territory, jurisdiction or area or of its authorities. This map is provided without any warranty of any kind, either express or implied.

Competing interests $\mathrm{JL}$ reported receiving research grants, through Fuwai Hospital, from the People's Republic of China for work to improve the management of hypertension and blood lipids and to improve care quality and patient outcomes of cardiovascular disease; receiving research agreements, through the National Center Centre for Cardiovascular Diseases and Fuwai Hospital, from Amgen for a multicenter clinical trial assessing the efficacy and safety of omecamtiv mecarbil and for dyslipidemic patient registration; receiving a research agreement, through Fuwai Hospital, from Sanofi for a multicentre clinical trial on the effects of sotagliflozin; receiving a research agreement, through Fuwai Hospital, with the University of Oxford for a multicenter clinical trial of empagliflozin; receiving a research agreement, through the National Centre for Cardiovascular Diseases, from AstraZeneca for clinical research methods training outside the submitted work; and receiving a research agreement, through the National Centre for Cardiovascular Diseases, from Lilly for physician training outside the submitted work. SSD reported receiving research funding from the US Food and Drug Administration (FDA), the National Evaluation System for Health Technology Coordinating Center (NESTCC), the National Heart, Lung, and Blood Institute (NHLBI) of the National Institutes of Health (NIH) (K12HL138046), the National Institute for Healthcare Management, Arnold Ventures, and the Greenwall Foundation.

Patient consent for publication Not applicable.

Ethics approval This study (No. 2015BAl12B02) was approved by the central ethics committee at Fuwai Hospital, Beijing, China (2016-752). Informed consent was waived because this study was based on retrospective record review. The central ethics committee at Fuwai Hospital approved the China PEACE Retrospective VHD Study. All collaborating hospitals accepted the central ethics approval except for 15 hospitals, which obtained local approval from internal ethics committees.

Provenance and peer review Not commissioned; externally peer reviewed

Data availability statement № data are available. Data are not available for sharing. All summary data relevant to the study are included in the article or uploaded as supplementary information.

Supplemental material This content has been supplied by the author(s). It has not been vetted by BMJ Publishing Group Limited (BMJ) and may not have been peer-reviewed. Any opinions or recommendations discussed are solely those of the author(s) and are not endorsed by BMJ. BMJ disclaims all liability and responsibility arising from any reliance placed on the content. Where the content includes any translated material, BMJ does not warrant the accuracy and reliability of the translations (including but not limited to local regulations, clinical guidelines, terminology, drug names and drug dosages), and is not responsible for any error and/or omissions arising from translation and adaptation or otherwise.

Open access This is an open access article distributed in accordance with the Creative Commons Attribution Non Commercial (CC BY-NC 4.0) license, which permits others to distribute, remix, adapt, build upon this work non-commercially, and license their derivative works on different terms, provided the original work is properly cited, appropriate credit is given, any changes made indicated, and the use is non-commercial. See: http://creativecommons.org/licenses/by-nc/4.0/.

\section{ORCID iDs}

Xinghe Huang http://orcid.org/0000-0002-3664-8779

Yuan Lu http://orcid.org/0000-0001-5264-2169

Harlan M Krumholz http://orcid.org/0000-0003-2046-127X

Jing Li http://orcid.org/0000-0002-8840-8638

\section{REFERENCES}

1 lung B, Vahanian A. Epidemiology of valvular heart disease in the adult. Nat Rev Cardiol 2011;8:162-72.

2 Yadgir S, Johnson CO, Aboyans V. Global burden of disease study 2017 nonrheumatic valve disease Collaborators. global, regional, and national burden of calcific aortic valve and degenerative mitral valve diseases, 1990-2017. Circulation 2020;141:1670-80.
3 Watkins DA, Johnson CO, Colquhoun SM, et al. Global, regional, and national burden of rheumatic heart disease, 1990-2015. N Engl J Med 2017;377:713-22.

4 d'Arcy JL, Prendergast BD, Chambers JB, et al. Valvular heart disease: the next cardiac epidemic. Heart 2011;97:91-3.

5 Zhu QF, Liu XB, Wang JA. Transcranial aortic valve replacement: current status, research progress and hot issues in 2016. Journal of Electrocardiology and Circulation 2016;35:387-9.

6 Liu F-Z, Xue Y-M, Liao H-T, et al. Five-Year epidemiological survey of valvular heart disease: changes in morbidity, etiological spectrum and management in a cardiovascular center of southern China. $J$ Thorac Dis 2014;6:1724-30.

7 Pan W, Zhou D, Cheng L, et al. Candidates for transcatheter aortic valve implantation may be fewer in China. Int $J$ Cardiol 2013;168:e133-4.

8 Cheng TO. How much of the recent decline in rheumatic heart disease in China can be explained by changes in cardiovascular risk factors? Int J Cardiol 2009;132:300-2.

9 Qi X, Xu H, Liu Q. Analysis of diagnosis and treatment status of hospitalized patients with degenerative heart valve disease in China. Chinese Circulation Journal 2019;34:771-6.

10 Shu C, Chen S, Qin T, et al. Prevalence and correlates of valvular heart diseases in the elderly population in Hubei, China. Sci Rep 2016;6:27253.

11 National Center for Cardiovascular Diseases, China. Annual report on cardiovascular health and diseases in China (2016). China: National Center for Cardiovascular Diseases, 2017.

12 Enriquez-Sarano M, Messika-Zeitoun D, Topilsky Y, et al. Tricuspid regurgitation is a public health crisis. Prog Cardiovasc Dis 2019;62:447-51.

13 Wang Y-T, Tao J, Maimaiti A, et al. Prevalence of valvular heart diseases and associated risk factors in Han, Uygur and Kazak population in Xinjiang, China. PLoS One 2017;12:e0174490.

14 Topilsky Y, Maltais S, Medina Inojosa J, et al. Burden of Tricuspid Regurgitation in Patients Diagnosed in the Community Setting. JACC Cardiovasc Imaging 2019;12:433-42.

15 Li J, Li X, Wang Q, et al. St-Segment elevation myocardial infarction in China from 2001 to 2011 (the China PEACE-Retrospective acute myocardial infarction study): a retrospective analysis of hospital data. The Lancet 2015;385:441-51.

16 Unger $\mathrm{P}$, Pibarot $\mathrm{P}$, Tribouilloy $\mathrm{C}$, et al. Multiple and mixed valvular heart diseases. Circ Cardiovasc Imaging 2018;11:e007862.

17 lung B, Baron G, Butchart EG, et al. A prospective survey of patients with valvular heart disease in Europe: the Euro heart survey on valvular heart disease. Eur Heart J 2003;24:1231-43.

18 Nishimura RA, Otto CM, Bonow RO. AHA/ACC guideline for the management of patients with valvular heart disease: Executive summary: a report of the American College of Cardiology/American heart association Task force on practice guidelines. J Am Coll Cardiol 20142014;63:2438-88.

19 Baumgartner H, Falk V, Bax JJ, et al. 2017 ESC/EACTS guidelines for the management of valvular heart disease. Eur Heart $J$ 2017;38:2739-91.

20 National Bureau of Statistics. China statistical Yearbook (2015). Beijing: China Statistics Press, 2016.

21 Hu P, Liu X-B, Liang J, et al. A hospital-based survey of patients with severe valvular heart disease in China. Int $J$ Cardiol 2017;231:244-7

22 lung B, Delgado V, Rosenhek R. Contemporary presentation and management of valvular heart disease: the EURObservational research programme valvular heart disease II survey. Circulation 2019;140:1156-69.

23 Jang SY, Ju E-Y, Seo SR, et al. Changes in the etiology of valvular heart disease in the rapidly aging Korean population. Int $J$ Cardiol 2014;174:355-9.

24 National Bureau of Statistics. China statistical Yearbook (2019. Beijing: China Statistics Press, 2020.

25 Arora S, Ramm CJ, Bahekar AA, et al. Evaluating Health of Emerging Economies Through the Eyes of Heart Valve Disease in the Transcatheter Era. Glob Heart 2017:12:301-4.

26 Remenyi B, EIGuindy A, Smith SC, et al. Valvular aspects of rheumatic heart disease. Lancet 2016;387:1335-46.

27 Liu S, Li Y, Zeng X, et al. Burden of cardiovascular diseases in China, 1990-2016: findings from the 2016 global burden of disease study. JAMA Cardiol 2019;4:342-52.

28 Topilsky Y, Nkomo VT, Vatury O, et al. Clinical outcome of isolated tricuspid regurgitation. JACC Cardiovasc Imaging 2014;7:1185-94.

29 Nath J, Foster E, Heidenreich PA. Impact of tricuspid regurgitation on long-term survival. J Am Coll Cardiol 2004;43:405-9.

30 Nkomo VT, Gardin JM, Skelton TN, et al. Burden of valvular heart diseases: a population-based study. Lancet 2006;368:1005-11. 
31 Rodés-Cabau J, Taramasso M, O'Gara PT. Diagnosis and treatment of tricuspid valve disease: current and future perspectives. Lancet 2016;388:2431-42.

32 Nishimura RA, Otto CM, Bonow RO, et al. 2014 AHA/ACC quideline for the management of patients with valvular heart disease: a report of the American College of Cardiology/American heart association Task force on practice guidelines. J Thorac Cardiovasc Surg 2014;148:e1-132.

33 Zhang $\mathrm{B}$, Xu H, Zhang $\mathrm{H}$, et al. Prognostic value of $\mathrm{N}$-terminal pro-Btype natriuretic peptide in elderly patients with valvular heart disease. J Am Coll Cardiol 2020;75:1659-72.

34 Dziadzko V, Clavel M-A, Dziadzko M, et al. Outcome and undertreatment of mitral regurgitation: a community cohort study. Lancet 2018;391:960-9.

35 Bach DS, Awais M, Gurm HS, et al. Failure of guideline adherence for intervention in patients with severe mitral regurgitation. J Am Coll Cardiol 2009;54:860-5.
36 Bach DS, Cimino N, Deeb GM. Unoperated patients with severe aortic stenosis. J Am Coll Cardiol 2007;50:2018-9.

37 Charlson E, Legedza ATR, Hamel MB. Decision-Making and outcomes in severe symptomatic aortic stenosis. J Heart Valve Dis 2006;15:312-21.

$38 \mathrm{Hu} \mathrm{K}, \mathrm{Li} \mathrm{J}$, Wan Y, et al. Heart valve disease in elderly Chinese population: effect of advanced age and comorbidities on treatment decision-making and outcomes. J Geriatr Cardiol 2016;13:593-601.

39 Triki F, Jdidi J, Abid D, et al. Characteristics, aetiological spectrum and management of valvular heart disease in a Tunisian cardiovascular centre. Arch Cardiovasc Dis 2017;110:439-46.

40 Aoyagi S, Tanaka K, Kosuga K, et al. Reoperation for acquired valvular heart disease--operative method and surgical results. Kurume Med J 1991;38:141-7.

41 Gao R. Current status of valvular heart disease in China. West China Medical Journal 2018;33:127-31. 